\title{
FINITE TYPE FUNCTIONS AS LIMITS OF EXPONENTIAL SUMS
}

\author{
D. J. NEWMAN ${ }^{1}$
}

\begin{abstract}
The functions $f(z)$ of type 1 which are bounded on the real axis are usually thought of as spanned by the exponentials $e^{i \lambda z}, \lambda \in[-1,1]$. We find the correct topology in which this "spanning" occurs.
\end{abstract}

The class of functions of finite exponential type, say type 1, which are bounded on the real axis is generally thought of as being "spanned" by the exponential functions $e^{i \lambda z}, \lambda \in[-1,1]$. Apparently, however, the "correct" topology for this spanning has not been recorded. Thus it is known that being of the form $\int_{-1}^{1} e^{i \lambda z} d \mu(\lambda)$ is sufficient for membership in the class, but not necessary, while being of the form $\lim _{n \rightarrow \infty} \int_{-1}^{1} e^{i \lambda z} d \mu_{n}(\lambda)$ is necessary, but not sufficient. We show that "weak" convergence is the proper topology.

DEFINITIONS. We say that a sequence of functions, $f_{n}(x)$, converges weakly to $f(x)$ or, in symbols, $f_{n}(x) \stackrel{w}{\rightarrow} f(x)$ when

(1) $f_{n}(x) \rightarrow f(x)$ uniformly on all finite intervals, and

(2) $\left|f_{n}(x)\right| \leqslant M, M$ fixed, for all $n$ and all real $x$.

The class $A_{1}$ consists of all functions which are weak limits of finite exponential sums, $\Sigma C_{\lambda} e^{i \lambda x}$ wherein all $\lambda$ lie in $[-1,1]$.

The class $B_{1}$ consists of all functions, $f(z)$, which are entire and satisfy the growth condition $|f(x+i y)| \leqslant M e^{|y|}, M$ fixed, for all real $x$ and $y$. (Equivalently $B_{1}$ is the class of type 1 functions which are bounded on the real axis.)

TheOREM. $A_{1}=B_{1}$.

Proof. I: $A_{1} \subseteq B_{1}$.

We need the following

LEMMA. Let $f(z)$ be analytic in $\operatorname{Im} z \geqslant 0$ and have its modulus bounded by 1 there. If $|f(z)| \leqslant \varepsilon$ on the interval $[-A, A]$ then $|f(z)| \leqslant \sqrt{\varepsilon}$ throughout the half disc $|z| \leqslant A$, $\operatorname{Im} z \geqslant 0$.

Proof. Fix $u+i v$ inside $|z| \leqslant A$, Im $z \geqslant 0$, and consider the linear fractional transformation $z \rightarrow u-v^{2} /(z-u)$. This is a map of $|z| \geqslant|u+i v|$ onto $|z| \leqslant \mid u+$ $i v \mid$ so that we always either have $|z| \leqslant|u+i v| \leqslant A$ or $\left|u-v^{2} /(z-u)\right| \leqslant|u+i v|$ $\leqslant A$. Hence if we form the auxiliary function $g(z)=f(z) f\left(u-v^{2} /(z-u)\right)$ we

Received by the editors February 8, 1982.

1980 Mathematics Subject Classification. Primary 30D15, 30D10.

${ }^{1}$ Sponsored by the United States Army under Contract No. DA-31-124-ARO-D-462.

(C1982 American Mathematical Society 0002-9939/82/0000-0647/\$01.75 
conclude that it is bounded by $1 \cdot \varepsilon$ on the real axis. Since it is clear that $g(z)$ is bounded, analytic in $\operatorname{Im} z \geqslant 0, z \neq u$ the maximum modulus theorem applies. Thus $|g(z)| \leqslant \varepsilon$ throughout $\operatorname{Im} z \geqslant 0$, and setting $z=u+i v$ gives $\left|(f(u+i v))^{2}\right| \leqslant \varepsilon$ which proves the lemma.

Now let $f_{n}(x) \stackrel{w}{\rightarrow} f(x)$ where each $f_{n} \in B_{1}$. Fix an $\varepsilon>0$ and an interval $[-A, A]$ and choose $m, n$ so large that $\left|f_{m}(x)-f_{n}(x)\right| \leqslant \varepsilon$ throughout $[-A, A]$. Since we also have $\left|f_{m}(x)-f_{n}(x)\right| \leqslant 2 M$ throughout $(-\infty, \infty)$ and since $\left(f_{m}(z)-f_{n}(z)\right) e^{i z}$ is bounded analytic in the upper half plane the lemma applies and gives

$$
\left|\left(f_{m}(z)-f_{n}(z)\right) e^{i z}\right| \leqslant \sqrt{2 M \varepsilon}
$$

throughout $|z| \leqslant A, \operatorname{Im} z \geqslant 0$. Thus we obtain $\left|f_{m}(z)-f_{n}(z)\right| \leqslant \sqrt{2 M \varepsilon} e^{A}$ throughout this upper half disc and in like manner it can be seen to hold in the lower half disc.

Therefore $f_{n}(z)$ converges uniformly on compact sets and so $\lim _{n} f_{n}(z)$ is entire. In other words $f(x)$ is (continuable to be) entire. Also since $\left|f_{n}(x+i y)\right| \leqslant M e^{|y|}$ we find that $|f(x+i y)| \leqslant M e^{|y|}$. In short $f \in B_{1}$.

All in all we have proven that $B_{1}$ is closed under taking weak limits and so, since each sum $\Sigma c_{\lambda} e^{i \lambda x},-1<\lambda<1$, is clearly in $B_{1}$, we conclude that $A_{1} \subseteq B_{1}$ as required.

II: $B_{1} \subseteq A_{1}$.

LEMMA. If $f(x) \in B_{1}$ and $K_{\varepsilon}(t)=(\cos t-\cos (1+\varepsilon) t) / \pi \varepsilon t^{2}, \varepsilon>0$, then we have, identically,

$$
f(x)=\int_{-\infty}^{\infty} f(x-t) K_{\varepsilon}(t) d t .
$$

Proof. Since $\int_{-\infty}^{\infty} K_{\varepsilon}(t) d t=1$ we may write this as

$$
0=\int_{-\infty}^{\infty}[f(x)-f(x-t)] K_{\varepsilon}(t) d t
$$

which is to say

$$
\begin{aligned}
0= & \int_{-\infty}^{\infty} \frac{f(x)-f(x-t)}{t} \frac{e^{i t}-e^{i(1+\varepsilon) t}}{t} d t \\
& +\int_{-\infty}^{\infty} \frac{f(x)-f(x-t)}{t} \frac{e^{-i t}-e^{-i(1+\varepsilon) t}}{t} d t
\end{aligned}
$$

Both of these integrals converge and we move the path of integration to $\operatorname{Im} t=A$ for the first integral and to $\operatorname{Im} t=-A$ for the second $(A>0)$. The first integral is estimated by

$$
\begin{aligned}
\int_{A i-\infty}^{A i+\infty} \frac{\left(M+M e^{A}\right)\left(e^{-A}+e^{-(1+\varepsilon) A}\right)}{|t|^{2}} d t & \leqslant \int_{A i-\infty}^{A i+\infty} \frac{2 M e^{A} \cdot 2 e^{-A}}{|t|^{2}} d t \\
& =4 M \int_{A i-\infty}^{A i+\infty} \frac{d t}{|t|^{2}}=\frac{4 M \pi}{A}
\end{aligned}
$$

and similarly for the second. Our identity therefore follows upon letting $A \rightarrow \infty$.

To prove II we use the fact that our weak convergence, for $B_{1}$ functions, is the same as the weak* convergence of the functions interpreted as linear functionals on 
$L^{1}(-\infty, \infty)$. Actually weak* convergence only implies pointwise, a.e., convergence rather than the locally uniform convergence we require, but for $B_{1}$ functions we have Bernstein's theorem that $\operatorname{Sup}\left|f^{\prime}(x)\right| \leqslant \operatorname{Sup}|f(x)|$ so that pointwise implies locally uniform convergence. This same reasoning allows us to equate the topology with sequential convergence.

Having identified weak convergence, then, as weak* convergence over $L^{1}$ we may deduce the "density" of the exponential polynomials by the standard functional analysis methods.

In short let $g(x)$ be any function in $L^{1}(-\infty, \infty)$ which satisfies $\int_{-\infty}^{\infty} g(x) e^{i \lambda x} d x=0$ for all $\lambda \in[-1,1]$. We need only show that, for any $f \in B_{1}, \int_{-\infty}^{\infty} g(x) f(x) d x=0$. To do so we consider $\int_{-\infty}^{\infty} g(x(1+\varepsilon)) f(x)_{\infty} d x$ and apply our identity. This gives

$$
\int_{-\infty}^{\infty} g(x(1+\varepsilon)) f(x) d x=\iint g(x(1+\varepsilon)) f(t) K_{\varepsilon}(x-t) d s d x
$$

and since $g \in L^{1}(d x), K \in L^{1}(d t)$, and $f$ is bounded, we may reverse the order of integration to conclude that this in turn equals

$$
\int f(t) \int g(x(1+\varepsilon)) K_{\varepsilon}(x-t) d x d t
$$

Next, by direct integration, we have

$$
K_{\varepsilon}(u)=\frac{1}{2 \pi} \int_{-(1+\varepsilon)}^{(1+\varepsilon)} \tau(S) e^{i S u} d S, \quad \tau(S)=1-\frac{(|S|-1)_{+}}{\varepsilon},
$$

so that our inner integral can be written

$$
\begin{aligned}
\frac{1}{2 \pi} \int_{-\infty}^{\infty} g(s(1+\varepsilon)) \int_{-(1+\varepsilon)}^{(1+\varepsilon)} \tau(S) e^{i S(x-t)} d s d x & \\
= & \frac{1}{2 \pi} \int_{-(1+\varepsilon)}^{(1+\varepsilon)} \tau(S) e^{-S t} \int_{-\infty}^{\infty} g(x(1+\varepsilon)) e^{i S x} d x d S
\end{aligned}
$$

but this inner integral is always 0 , by hypothesis. We have proven, then, that $\int_{-\infty}^{\infty} f(x) g(x(1+\varepsilon)) d x=0$, or $\int_{-\infty}^{\infty} f(x /(1+\varepsilon)) g(x) d x=0$. Letting $\varepsilon \rightarrow 0$ and using the dominated convergence theorem we obtain, finally $\int_{-\infty}^{\infty} f(x) g(x) d x=0$ and our proof is complete.

Department of Mathematics, Temple University, Philadelphia, Pennsylvania 19122 